\title{
Tomographic diffractive microscopy: principles and applications
}

\section{Olivier Haeberlé}

Olivier Haeberlé, "Tomographic diffractive microscopy: principles and applications," Proc. SPIE 10677, Unconventional Optical Imaging, 106770J (24 May 2018); doi: 10.1117/12.2500236

SPIE. Event: SPIE Photonics Europe, 2018, Strasbourg, France 


\title{
Tomographic diffractive microscopy: principles and applications
}

\author{
Olivier Haeberlé \\ IRIMAS EA7499, Université de Haute-Alsace, Mulhouse, France
}

\begin{abstract}
Tomographic Diffractive Microscopy (TDM) is an advanced digital imaging technique, extending the concept of Digital Holographic Microscopy (DHM), which provides quantitative information about the index of refraction distribution within the observed sample, by recording of multiple holograms under varying conditions of illumination, then applying numerical inversion procedures to reconstruct a 3-D image of the specimen under consideration. After a short recall of DHM applications and limitations, various implementations of TDM are described, highlighting their respective advantages and. drawbacks. To conclude, some perspectives and challenges for this imaging modality are presented.
\end{abstract}

Keywords: Digital Holographic Microscopy; Tomographic Diffractive Microscopy; Computational Imaging; Diffraction

\section{INTRODUCTION}

Its unique capabilities for imaging living specimens, in three dimensions, possibly over long periods make the optical microscope an invaluable tool for biological research. Fluorescence microscopy is the reference method, because it allows for specific labeling of cellular structures and functions, and thanks to the unsurpassed resolution obtainable with modern techniques like STED or PALM/STORM. Fluorescence labeling may however induce unfavorable effects in some cases, like photo-toxicity, and labeling itself may be difficult. Speed is also rather limited, especially for 3-D imaging. In some cases, use of fluorescent markers is also to be avoided. This explains that in recent years, there has been a regain of interest for developing new imaging techniques not requiring sample labeling. In particular, the coupling of fast-, high-sensitivity and high-resolution electronic sensors with the exploding capacities of recent computers has open new possibilities for revisiting optical microscopy.

\section{DIGITIAL HOLOGRAPHIC MICROSCOPY}

In conventional transmission microscopy (classical wide-field, dark-field, phase-contrast, Differential Interference Contrast, Hoffman modulation, etc), the image is formed by a complex interaction of the incoherent illuminating light with the specimen. The recorded image is an intensity-only image, which contrast is very efficient to detect structures, and therefore is for example very helpful for morphological evolution studies, but which does not easily deliver quantitative information on the optical characteristics of the observed specimen. In particular, phase and absorption properties mix to form the final image, and a darker, therefore apparently absorbing zone may in fact simply results from a destructive interference phenomenon.

On the contrary, the use of coherent light illumination, combined with interferometric detection, permits to record holograms, encoding both the amplitude and phase of the light interacting with the specimen, therefore delivering a more complete information about the diffraction phenomenon. Wavefront analyzers also permit to record the amplitude and phase of the diffracted light, without the need of an interferometer, and with short coherence illumination. These approaches, usually coined as Digital Holographic Microscopy or phase microscopy [1]-[4], have in particular proven to be very sensitive to small sample changes, thanks to unsurpassed precision of interferometric measurements. Several commercial implementations are now available, and phase imaging is now used for many biological studies, including red blood cells investigation (e.g. malaria infection), non-contact neuronal activity measurements, long period time-lapse recording of cell growth or cell dynamics, high-throughput screening for drug testing, etc.

But while DHM is very powerful considering integral measurements (projections), its main drawback is its poor resolution when reconstructing 3-D images of transparent specimens, especially along the optical axis. This limitation is easily understood when remembering that only one illumination direction is used (contrary to classical microscopy using an illumination condenser), translating into limited quantity of recorded information [5], so poor 3-D image reconstructions. 


\section{TDM WITH ILLUMINATION ROTATION}

We consider an object defined by its relative permittivity $\varepsilon(\mathrm{r})$ and illuminated by a scalar monochromatic incident wave,

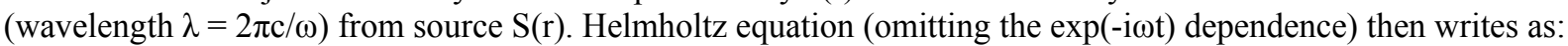

$$
\Delta E(\mathbf{r})+k_{0}^{2} E(\mathbf{r})=X(\mathbf{r}) k_{0}^{2} E(\mathbf{r})+S(\mathbf{r})
$$

with $\mathrm{k}_{0}=2 \pi / \lambda$, and $X=1-\varepsilon$ being the contrast of permittivity. With the Green function:

$$
\mathrm{G}(\mathrm{r})=-\exp \left(\mathrm{ik}_{0} \mathrm{r}\right) / 4 \pi \mathrm{r},
$$

one obtains the integral equation for the total field:

$$
E(\mathbf{r})=E_{\text {inc }}(\mathbf{r})+k_{0}^{2} \int G\left(\mathbf{r}-\mathbf{r}^{\prime}\right) X\left(\mathbf{r}^{\prime}\right) E\left(\mathbf{r}^{\prime}\right) d \mathbf{r}^{\prime}
$$

with $\mathrm{E}_{\text {inc }}$ being the field that would exist in the absence of the object. The integral in (3) restricts to support $\Omega$ of the object. For an observation point $r$ far from the object, so that $r^{\prime 2} / \lambda<<$, with $r^{\prime}$ in $\Omega$, the diffracted field simplifies as:

$$
E_{d}(\mathbf{r})=-\frac{\exp \left(i k_{0} r\right)}{4 \pi r} e(\mathbf{k})
$$

with the scattered far-field amplitude written as:

$$
e(\mathbf{k})=k_{0}^{2} \int \exp \left(-i \mathbf{k} . \mathbf{r}^{\prime}\right) X\left(\mathbf{r}^{\prime}\right) E\left(\mathbf{r}^{\prime}\right) d \mathbf{r}^{\prime}
$$

Considering a plane wave with wavevector $\mathrm{k}_{\text {inc }}$ as incident field: $\mathrm{E}_{\text {inc }}(\mathrm{r})=\mathrm{A}_{\text {inc }} \exp \left(\mathrm{ik} \mathrm{inc}_{\mathrm{inc}} \cdot \mathrm{r}\right)$, and for a weakly diffracting object, for which the first Born approximation is valid [2], one finally obtains:

$$
e\left(\mathbf{k}, \mathbf{k}_{i n c}\right)=C \widetilde{X}\left(\mathbf{k}-\mathbf{k}_{i n c}\right)
$$

where $\mathrm{C}=8 \pi^{3} \mathrm{~A}_{\text {inc }} \mathrm{k}_{0}^{2}$. Equation (6) provides a direct correspondence between the diffracted far-field amplitude and the Fourier coefficient of the relative permittivity of the object. Energy conservation in the diffraction process implies that diffracted $\mathbf{k}_{\text {diff }}$ vectors have same amplitude as $\mathbf{k}_{\text {inc }}$, and therefore depict the so-called Ewald sphere. Because of the limited numerical aperture of the detection objective, only a cap of the Ewald sphere can be captured. Considering elastic scattering only, this set of diffracted $\mathbf{k}_{\text {diff }}$ vectors then transforms into object $\mathbf{k}_{\mathrm{o}}$ vectors, via a simple translation:

$$
\mathbf{k}_{\mathrm{o}}=\mathbf{k}_{\mathrm{diff}}-\mathbf{k}_{\mathrm{inc}}
$$
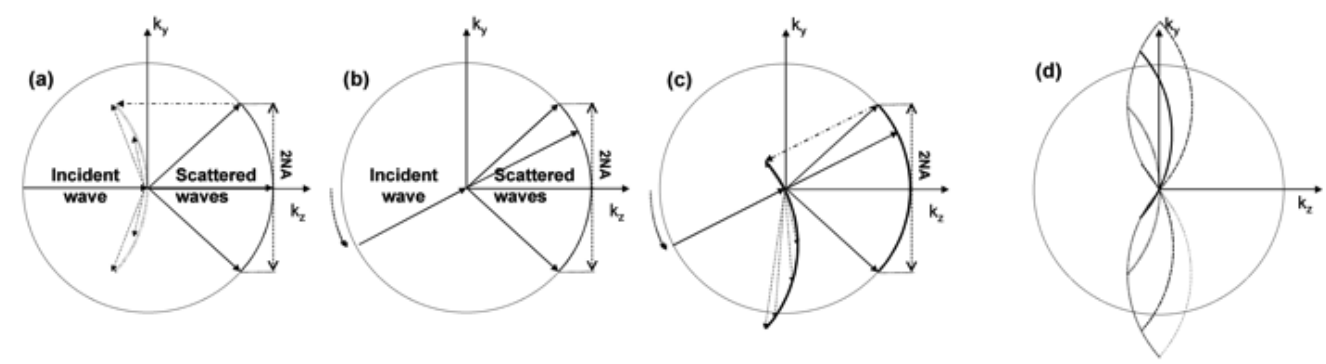

Figure 1. Principle of information acquisition in $\mathrm{k}$ vector space for (a): Digital Holographic Microscopy with one illumination, and (b)-(d): Tomographic Diffractive Microscopy with varying illumination angle.

Figure 1 depicts the process of information acquisition for DHM (Fig. 1(a)), and for TDM with illumination rotation (Figs. 1(b)-(d)). From Fig. 1(a), one understands that the lateral extension of the $\mathbf{k}_{\mathrm{o}}$ vector set will provide good lateral resolution in image space. In contrary, the narrow extension along the $\mathrm{k}_{\mathrm{z}}$ axis translates into very poor resolution and optical sectioning along the corresponding optical axis [5].

So, in order to improve image quality, one has to expand the $\mathbf{k}_{\mathrm{o}}$ coverage. In TDM with illumination rotation, the illumination can be tilted (Fig. 1(b)), so that one finally records a complementary subset of information (Fig. 1(c)). For a large number of illuminations, a synthetic aperture approach delivers an enlarged frequency support (Fig. 1(d)). The latter explains the better resolution and optical sectioning capability of TDM; compared to DHM. On can theoretically double the lateral resolution [6][7], experimentally reaching resolution in the $100 \mathrm{~nm}$ range [8][9]. Also, it allows for a 
clear distinction of refractive and absorptive subregions within the observed sample [10]. This technique is developed in several laboratories, and now even available commercially [11][12]. See websites of Nanolive [11] and Tomocube [12] companies for various examples of applications on biological samples. The Nanolive system offers a large accessible space over the sample, while the Tomocube allows for faster acquisitions.

\section{MULTIWAVELENGTH TDM}

A variant of TDM for increasing the frequency coverage in Fourier space consists in varying the illumination wavelength. Figure 2(a) describes the obtained object frequency support, for two wavelengths $\lambda_{1}$ and $\lambda_{2}=2 \lambda_{1}$. Both caps of sphere (arc of circles in 2-D) have their summit at the frequency origin, so that a continuous wavelength scanning could provide a filled frequency support in-between (Fig. 2(b)).

The main advantage of this approach is that a no-moving-part setup could be built, contrary to TDM with illumination rotation or with sample rotation, but one can also see that it gives access to a more limited frequency range. Furthermore, wide spectrum ("white") coherent light sources are still of limited availability, and complex.

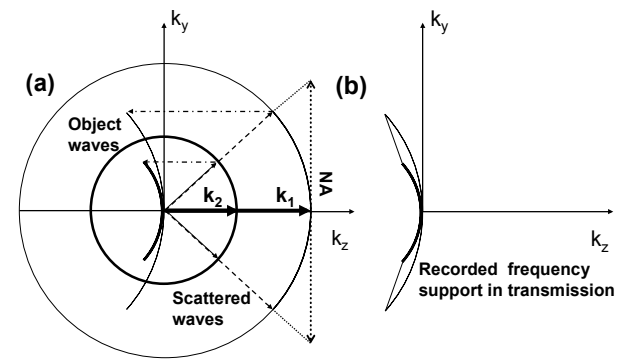

Figure 2. TDM with illumination wavelength variation: construction of the object frequency support

This problem has been nicely solved by the introduction of white-light diffraction tomography [13][14], which can be seen as an extension of phase contrast microscopy, with controllable phase masks. Scanning the observed sample in $z$, and properly processing of the data then allows for reconstruction of 3-D transparent samples in a tomographic mode. This technique is also commercially distributed [15], with the advantage of being available as an add-on module, which can be fitted to a standard microscope. The PhiOptics website describes various applications of this technique.

\section{TDM WITH SPECIMEN ROTATION}

Another method to increase captured object frequencies coverage consists in rotating the sample, while keeping the illumination and detection static. Figure 3 describes the obtained object frequency support. This approach delivers quasiisotropic resolution images, with only a small, so-called "missing apple-core", along the specimen rotation axis [16].

The main advantage of this technique is that a standard DHM can be used, but performing precise sample rotation, compatible with interferometric measurements for TDM reconstructions, is challenging. Sample can be embedded within a rotating microcapillary [17][18]. Recently, optical tweezers [19][20] and dielectrophoretic cell rotation [21] have been used, as well as natural rotation of samples such as red blood cells when flowing through microfluidic channels [22].

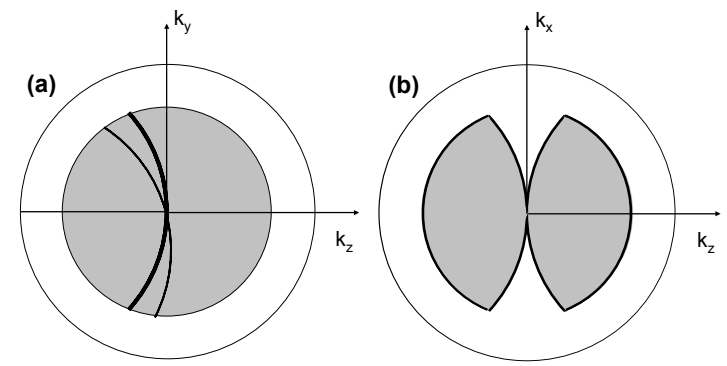

Figure 3. Object frequency support for TDM with specimen rotation. (a): in $\mathrm{k}_{\mathrm{y}}-\mathrm{k}_{\mathrm{z}}$ plane. Thick solid line: frequency support obtained for the initial position of the sample, thin solid line: frequency support obtained for one sample rotation around the $\mathrm{x}$-axis. Grey disk: frequency support obtained when the sample is continuously rotated. (c): as (a) but in the $\mathrm{k}_{\mathrm{x}}-\mathrm{k}_{\mathrm{z}}$ plane. 


\section{COMBINED APPROCHES}

As TDM with illumination rotation and TDM with sample rotation both present sets of uncaptured frequencies, but oriented along different axes (z-axis, or optical axis for TDM with illumination rotation, and sample rotation axis for TDM with sample rotation), which are perpendicular to each other, combining both approaches has been proposed [23], in order to obtain simultaneously improved- and isotropic-resolution images. Figure 4 describes the obtained frequency support for (a): one acquisition with TDM with illumination rotation, (b): two acquisitions with one $90^{\circ}$ sample rotation, (c): 4 acquisitions with $45^{\circ}$ sample rotations. This method has for advantage, compared to TDM with sample rotation only (Fig. 3), that only a few rotations are necessary to properly fill the Fourier space. This approach has been successfully demonstrated, delivering high-resolution images of test samples such as optical fiber tip, microcrystals or pollens [24]. Recently, a variant involving full sample rotation TDM acquisition (using optical tweezers) with data from TDM with illumination rotation has also been published [25], highlighting the possibility of studying free-floating live yeasts with this approach.
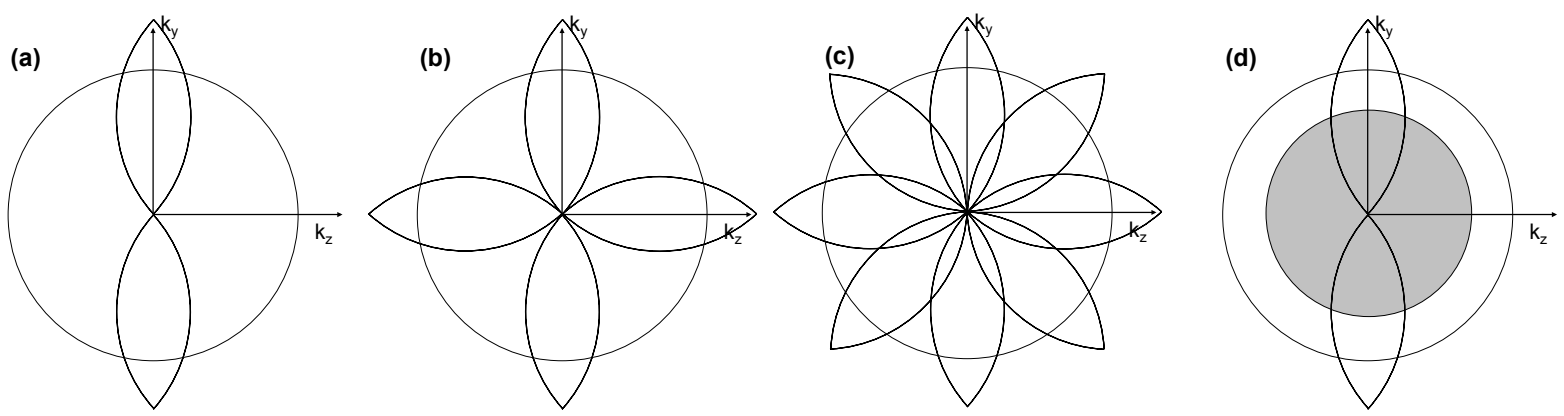

Figure 4. Combined tomography, with illumination and specimen rotation. (a)-(b)-(c): TDM with illumination rotation acquisitions for 1, 2 and 4 sample positions, respectively. (d): Combination of one TDM with illumination rotation acquisition and one TDM with sample rotation (in grey) acquisition.

\section{CONCLUSION AND PERSPECTIVES}

Tomographic diffractive microscopy allows for high-resolution observations of unlabeled samples, with various implementations, attracting the interest of more and more end-users. Its sequential nature is among present limitations, because it limits speed acquisition. Also, its computational nature limits final 3-D image delivery rate. But high-speed acquisitions are possible [26], and modern GPUs also allow for high-speed reconstructions [27], the present bottleneck being the data transfer rate between camera and computer. Several groups are also active in developing simplified, lowcost approaches [28][29], and TDM in reflection mode, not developed in this summary, also present advantages compared to standard optical microscopy, even with simple image reconstruction approaches [30]. Note also that a close relationship does exist between conventional microscopy and TDM [7], and recently, a unified description of TDM has been proposed, to which the interested reader is referred [31] for more details.

\section{REFERENCES}

[1] Kim, M. K., Digital Holographic Microscopy: Principles, Techniques, and Applications, Springer Series in Optical Science Vol. 162, Springer (2011)

[2] Popescu, G., Quantitative Phase Imaging of Cells and Tissues, McGraw Hill (2011)

[3] Biomedical Optical Phase Microscopy and Nanoscopy, Edited by N. T. Shaked, Z. Zalevsky, and L. Satterwhite, Elsevier (2012)

[4] Kemper B., Digital holographic microscopy: quantitative phase imaging and applications in live cell analysis, in [Handbook of Coherent-Domain Optical Methods], V. V. Tuchin, ed., Springer, 215-257 (2013).

[5] Debailleul, M., Simon, B., Georges, V., Haeberlé, O., and Lauer V., "Holographic microscopy and diffractive microtomography of transparent samples," Meas. Sci. Technol. 19, 074009 (2008).

[6] Lauer, V., "New approach to optical diffraction tomography yielding a vector equation of diffraction tomography and a novel tomographic microscope", J. Microscopy 205, 165-176 (2002) 
[7] Haeberlé, O., Belkebir, K., Giovannini, H., and Sentenac, A., "Tomographic Diffractive Microscopy: Basics, Techniques and Perspectives", J. Mod. Opt. 57, 686-699 (2010)

[8] Debailleul, M., Georges, V., Simon, B., Morin, R., and Haeberlé, O., "High resolution three-dimensional tomographic diffractive microscopy of transparent inorganic and biological samples", Opt. Lett. 34, 79-81 (2009)

[9] Cotte, Y., Toy, F., Jourdain, P., Pavillon, N., Boss, D., Magistretti, P., Marquet, P., and Depeursinge, C., "Marker-free phase nanoscopy", Nature Phot. 7, 113-117 (2013)

[10] Simon, B., Debailleul, M., Beghin, A., Tourneur, Y., and Haeberlé, O., "High resolution tomographic diffractive microscopy of biological samples," J. Biophotonics 3, 462-467 (2010)

[11] http://nanolive.ch

[12] http://www.tomocube.com

[13] Wang, Z., Marks, D. L., Carney, P. S., Millet, L. J., Gillett, M. U., Mihi, A., Braun, P. V., Shen, Z., Prasanth, S. G., and Popescu, G., "Spatial Light Interference Tomography (SLIT)", Opt. Exp. 19, 19907-19918 (2011)

[14] Kim, T., Zhou, R., Mir, M., Babacan, S. D., Scott Carney, P., Goddard, L. L., and Popescu, G., "White-light diffraction tomography of unlabelled live cells", Nature Photonics 8, 256-263 (2014)

[15] http://phioptics.com

[16] Vertu, S., Delaunay, J.-J., Yamada, I., and Haeberlé, O., "Diffraction microtomography with sample rotation: influence of a missing apple core in the recorded frequency space", Centr. Eur. J. of Phys. 7, 22-31 (2007)

[17] Kujawińska, M., Krauze, W., Kus, A., Kostencka, J., Kozacki, T., Kemper, B., and Dudek, M., "Problems and Solutions in 3-D Analysis of Phase Biological Objects by Optical Diffraction Tomography", Int. J. Optomechatronics 8, 357-372 (2014)

[18] Kuś, A., Dudek, M., Kemper, B., Kujawińska, M., Vollmer, A., "Tomographic phase microscopy of living three-dimensional cell cultures", J. Biomed. Opt 19, 4600 (2014)

[19] Habaza, M., Gilboa, B., Roichman, Y., and Shaked N. T., "Tomographic phase microscopy with $180^{\circ}$ rotation of live cells in suspension by holographic optical tweezers", Opt. Lett. 40, 1881-1884 (2015)

[20]Lin, Y.-C., Chen, H.-C., Tu, H.-Y., Liu, C.-Y., and Cheng, C.-J., "Optically driven full-angle sample rotation for tomographic imaging in digital holographic microscopy" Opt. Lett. 42, 1321-1324 (2017)

[21] Habaza, M., Kirschbaum, M., Guernth-Marschner, C., Dardikman, G., Barnea, I., Korenstein, R., Duschl, C., and Shaked, N. T. "Rapid 3D Refractive-Index Imaging of Live Cells in Suspension without Labeling Using Dielectrophoretic Cell Rotation", Adv. Sci. 4, 1600205 (2017)

[22] Merola, F., Memmolo, P., Miccio, L., Savoia, R., Mugnano, M., Fontana, A., D'Ippolito, G., Sardo, A., Iolascon, A. Gambale, A. Ferraro, P., "Tomographic flow cytometry by digital holography", Light: Science \& Applications 6, e16241 (2017)

[23] Vertu, S., Flügge, J., Delaunay, J.-J., and Haeberlé, O., "Improved and isotropic resolution in tomographic diffractive microscopy combining sample and illumination rotation", Centr. Eur. J. of Phys. 9, 969-974 (2011)

[24] Simon, B., Debailleul, M., Houkal, M., Ecoffet, C., Bailleul, J., Lambert, J., Spangenberg, A., Liu, H., Soppera, O., and Haeberlé, O., "Tomographic diffractive microscopy with isotropic resolution", Optica 4, 460-463 (2017)

[25] Vinoth B., Lai, X.-J., Lin, Y.-C., Tu, H.-Y., and Cheng, C.-J., "Integrated dual-tomography for refractive index analysis of free-floating single living cell with isotropic superresolution", Scientific Reports 8, 5943 (2018)

[26] Kim, M., Choi, Y., Fang-Yen, C., Sung, Y., Dasari, R. R., Feld, M. S., and Choi, W., "High-speed synthetic aperture microscopy for live cell imaging" Opt. Lett. 36, 148-150 (2011)

[27] Bailleul, J., Simon, B., Debailleul, M., Foucault, L., Verrier, N., and Haeberlé, O., "Tomographic diffractive microscopy: towards high-resolution 3-D real-time data acquisition, image reconstruction and display of unlabeled samples", Opt. Comm (accepted, to appear 2018) DOI: 10.1016/j.optcom.2017.11.008

[28] Isikman, S. O., Bishara, W., Mavandadi, S., Yu, F. W., Feng, S., Lau, R., and Ozcan, A., "Lens-free optical tomographic microscope with a large imaging volume on a chip", PNAS 1015638108 (2011)

[29] Berdeu, A., Momey, F., Laperrousaz, B., Bordy, T., Gidrol, X., Dinten, J.-M., Picollet-D'hahan, N., and Allier, C., "Comparative study of fully three-dimensional reconstruction algorithms for lens-free microscopy", Appl. Opt. 56, 3939-3951 (2017)

[30]Liu, H., Bailleul, J., Simon, B., Debailleul, M., Colicchio, B., and Haeberlé, O., "Tomographic diffractive microscopy and multiview profilometry with flexible aberration correction" Appl. Opt. 53, 755 (2014)

[31] Sentenac, A., and Mertz, J., "Unified description of three-dimensional optical diffraction microscopy: from transmission microscopy to optical coherence tomography: tutorial", J. Opt. Soc. Am. A 35, 748-754 (2018) 